\title{
Espalhamento de Transientes Eletromagnéticos por Cilindros Condutores de Seções Retas Arbitrárias
}

\author{
Carlos Leonidas S. S. Sobrinho e Emanoel Costa
}

\begin{abstract}
O espalhamento de transientes eletromagnéticos por cilindros condutores perfeitos de seçōes retas arbitrárias foi estudado numericamente nos domínios do tempo e da frequência, para os modos TE e TM, utilizando-se o método dos momentos. Mostrou-se que o tempo de processamento necessário para a solução do problema no domínio da frequência é inferior ao seu equivalente no domínio do tempo, principalmente quando técnicas espeć́ficas são adicionalmente empregadas. Os campos espalhados por três estruturas foram analisados, tendo os resultados obtidos mostrado boa concordância com os existentes na literatura.
\end{abstract}

\section{Introdução}

Pretende-se, como objetivo principal, comparar diversas técnicas de cálculo de campos espalhados por um cilindro infinito condutor perfeito de seção reta arbitrária, em resposta a um pulso eletromagnético estreito incidente sobre o mesmo. Desta maneira, será selecionada a mais eficiente em termos de área e tempo de processamento, assim como de precisāo de cálculo.

O estudo desta classe de problemas encontra aplicaçōes nas áreas de identificaçăo de objetos (espalhamento inverso) e compatibilidade eletromagnética. Na primeira, deseja-se determinar características do objeto a partir de informaçōes relativas aos sinais incidente e espalhado, supostos conhecidos. $\mathrm{Na}$ segunda, deseja-se analisar os efeitos de sinais do tipo impulsivo, naturais ou artificiais, sobre equipamentos eletrônicos.

Para condutores de formas arbitrárias, métodos aproximados têm sido usados com sucesso para determinar distribuiçōes de correntes e campos

C. L. S. Souza Sobrinho é Professor do Departamento de Engenharia Elétrica da Universidade Federal do Pará, Campus Universitário do Guamá - Centro Tecnológico, 66.050, Belém, PA.

E. Costa é Professor do Centro de Estudos em Telecomunicaçōes, PUC/RIO,

Rua Marquês de São Vicente 225, 22.453, Rio de Janeiro, RJ 
espalhados. Para espaihadores de pequenas dimensóes (em relação ao comprimento de onda), a teoria clássica do espalhamento Rayleigh [1] pode ser aplicada. No limite oposto, o problema tem sido resolvido através de métodos assintóticos (ótica geométrica, ótica física e teoria geométrica da difração) [2] e [3].

Para os espalhadores de dimensōes intermediárias estudados no presente trabalho, nenhum dos métodos acima produzirá um resultado totalmente satisfatório. Neste regime, resoluçōes numéricas de soluçōes formais se impóem. Uma vertente importante destas técnicas baseia-se na resoluçáo das formas diferenciais das equaçōes de Maxwell pelo método das diferenças finitas. Estas, entretanto, não são as mais apropriadas ao tratamento do presente problema [4] e [5]. A análise do espalhamento devido a estruturas impenetráveis, cujas superfícies têm formas de complexidade moderada, é realizada mais eficientemente pela soluçāo de equaçōes integrais com o auxílio do método dos momentos. Diversos conjuntos de equaçōes integrais resolvem formalmente o mesmo problema de espalhamento. Deduçóes destas equações a partir de resultados mais básicos, juntamente com discussōes detalhadas das opçóes existentes, podem ser encontradas na literatura [6]-[11]. Por economia de espaço, seräo omitidas. A necessidade de se solucionar numericamente estas equaçóes pode tornar um dado conjunto mais adequado ao tratamento de um problema espećfico. Somente as equaçōes integrais do campo magnético (EICM) para os modos transversais elétrico (TE) e magnético (TM), tanto no domínio da frequência quanto do tempo, serāo utilizadas aqui. As referências citadas imediatamente acima indicam serem estas apropriadas ao tratamento do problema a ser estudado.

Desta forma, estas equaçóes integrais serăo apenas apresentadas, evidenciando-se suas características gerais. Em seguida, apresentar-se-á a aplicação do método dos momentos [11] à soluçāo das equaçóes obtidas no domínio da frequência e ao cálculo do campo transiente espalhado. Ver-se-á, ainda, uma interpretaçáo do algoritmo proposto por Bennet e Weeks [12] para a so uçāo das equaçóes integrais no domínio do tempo, em termos do mesmo método.

- método dos momentos transforma as equaçóes integrais em um sistema ze ezuaçóes lineares. A maior parte dos recursos computacionais necessá- is cara a resoluçāo das equções integrais é utilizada no cálculo e armaze- $=-$ e - $:$ dos coeficientes do sistema e na sua soluçāo. Esta será obtida de

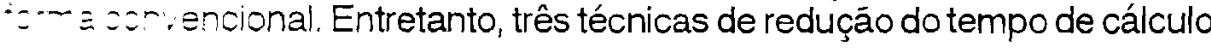


dos coeficientes do sistema linear serāo apresentadas. A primeira deve, necessariamente, ser utilizada, sendo as demais de uso opcional.

Em seguida, será realizada uma análise dos resultados obtidos para diversas estruturas. Será, ainda, apresentada uma análise comparativa das diversas técnicas de cálculo. Observou-se que os cálculos nos domínios da frequência e do tempo necessitam de áreas de processamento aproximadamente iguais. Entretanto, o tempo de processamento no domínio da frequência é nitidamente inferior ao do domínio do tempo, mesmo quando as técnicas opcionais mencionadas acima náo sāo utilizadas.

Em todas as situaçóes analisadas, observou-se a superioridade de uma destas técnicas opcionais (sempre a mesma) em relaçāo à outra.

\section{Equações Integrais Usadas nos Cálculos das Correntes e dos Campos Espalhados}

Seja um cilindro infinito condutor perfeito de seção reta arbitrária, imerso no espaço livre. Suponha um pulso Gaussiano plano que se propaga numa direçăo qualquer ortogonal ao eixo do cilindro e incide sobre o mesmo. Deseja-se determinar o campo espalhado em um ponto de observaçāo qualquer, distante do cilindro.

A soluçāo formal deste problema usando equaçōes integrais, principalmente quando se supōem variaçóes harmônicas no tempo $\left(e^{i \omega t}\right)$ para os campos e correntes, é bem conhecida [6]-[11]. Em particular, sabe-se que o problema vetorial inicial pode ser decomposto em dois modos escalares, que podem ser tratados separadamente. Sem perda de generalidade, pode-se introduzir um sistema de coordenadas retangulares, de forma que a direção de incidência e o eixo do cilindro estejam alinhados com os eixos $x$ e $z$, respectivamente. Para o modo transversal elétrico (TE), os campos magnéticos incidente e espalhado, assim como a corrente no cilindro, estarăo contidos no plano transversal (xy), conforme mostra a Fig. 1.

Para o modo transversal magnético (TM), os campos elétricos incidente e espalhado, assim como a corrente no cilindro, terāo sempre a direção z e os campos magnéticos incidente e espalhado estarāo contidos no plano transversal, conforme mostra a mesma figura. 


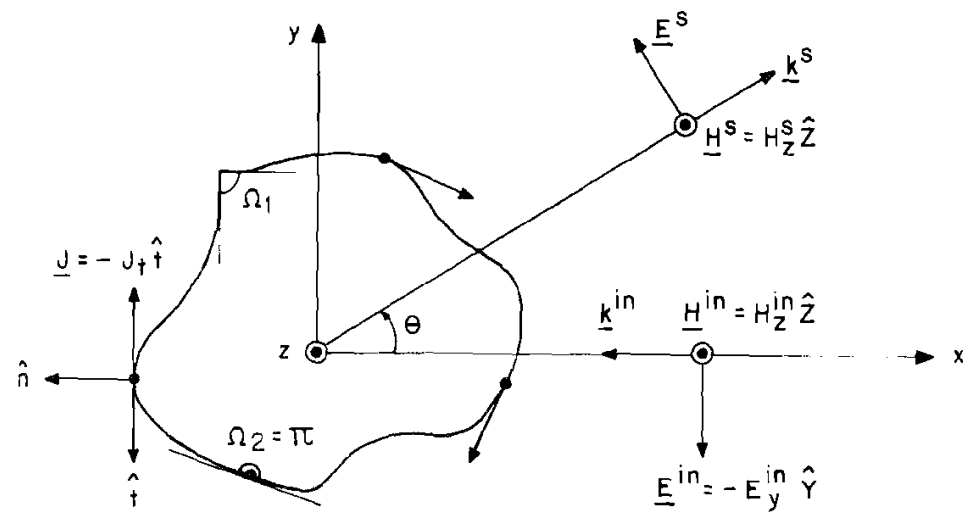

(o) MODO TE

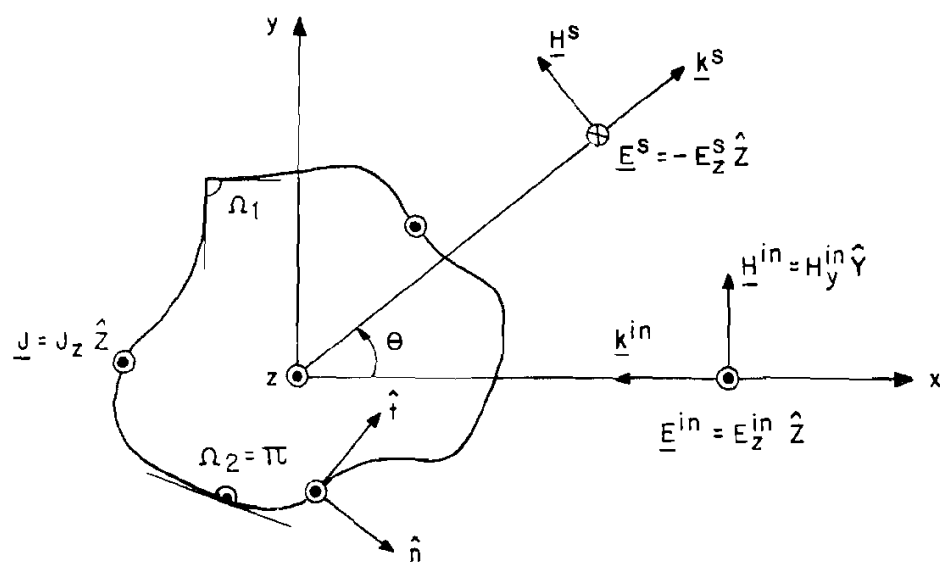

(b) MODO TM

Figura 1. Representação dos vetores campos incidente e espalhado e densidade de corrente para os modos TE e TM.

\subsection{Equaçóes Integrais para a Determinaçáo das Correntes no Cilindro}

No domínio da frequencia (DF), cada componente da densidade de corrente se relaciona à respectiva (para o mesmo modo) componente do campo magnético incidente tangencial à superfície do cilindro num dado ponto de observaçāo através da equaçăo (EICM-DF) [6], [8] e [11] 


$$
J(\mathbf{r}, \omega)=\zeta T H^{\text {in }}(\mathbf{r}, \omega)-i \frac{T \omega}{4 c} \oint_{C}(\hat{\eta}, \hat{p}) H_{1}^{(2)}\left(\frac{\omega}{c} \mid \mathbf{r}-\mathbf{r}^{\prime} l\right) J\left(\mathbf{r}^{\prime}, \omega\right) d l^{\prime}
$$

Nesta equaçāo, $J(\mathbf{r}, \omega)$ e $H^{\text {in }}(\mathbf{r}, \omega)$ representam: (i) para o modo TE, as componentes transversal $J_{t}(r, \omega)$ da densidade de corrente e axial $H_{z}^{\text {in }}(\mathbf{r}, \omega)$ do campo magnético incidente, respectivamente: (ii) para o modo $T M$, as componentes axial $J_{z}(r, \omega)$ da densidade de corrente e transversal $H_{t}^{\text {in }}(\mathbf{r}, \omega)$ do campo magnético incidente, respectivamente. Adicionalmente, $\omega$ é a frequência angular, $r$ e $r$ representam os vetores posiçōes dos pontos de observaçāo e "fonte", respectivamente, ambos na superfície do cilindro. $T=(1-\Omega / 2 \pi)^{-1}$, onde $\Omega$ representa o ângulo interno subentendido pela seçāo reta da superfície no ponto $r$, conforme mostra a Fig. 1. Em particular, $\Omega=\pi$ para pontos definidos em regiōes suaves da seção reta. Tem-se, ainda, que, para o modo $T E\{T M\}, \mu$ é o vetor unitário normal ao contorno $C$ que limița a seção reta e dirigido para fora do cilindro, no ponto "fonte" $\mathbf{r}^{\prime}\left(\mu=n^{\prime}\right)\{$ de observação $\mathbf{r}(\mu=\hat{n})\}$ e $\xi=-1\{1\}$. O vetor $\hat{p}$ é unitário na direçāo do vetor $\boldsymbol{p}=\left(\mathbf{r}-\mathbf{r}^{\prime}\right)$ e $\mu$. p representa o produto escalar entre os dois vetores. Ainda nesta expressāo, $H_{1}^{(2)}(s)$ representa a funçāo de Hankel do segundo tipo, primeira ordem e argumento s. As constantes ce i representam a velocidade da luz no vácuo e o imaginário puro. Finalmente, deve-se observar que a integral ao longo do contorno $C$ deve ser realizada no sentido do valor principal de Cauchy quando $\mathbf{r}$ 'tende a $\mathbf{r}$ (indicado pelo traço horizontal).

As componentes $H^{\text {in }}(\mathbf{r}, \omega)$ do campo magnético incidente tangenciais à superfície do cilindro no ponto $r$ podem ser representadas através da seguinte expressão geral

$$
H^{i n}(\mathbf{r}, \omega)=n_{x} e^{i \frac{\omega}{c}\left(x-x_{0}\right)} e^{-\left(\frac{\omega}{2 G}\right)^{2}}
$$

Q parâmetro $n_{x}$ é igual a 1 para o modo TE e igual à componente $x$ do vetor $n$ para o modo TM. A primeira exponencial mostra a variação da fase do campo incidente com a frequência e a abscissa $x$ do ponto de observaçāo (a partir de um plano $x=x_{0}$ arbitrário). A segunda exponencial mostra a dependência da amplitude do campo incidente com a frequência (segundo uma funçāo Gaussiana de largura determinada pelo parâmetro G). 
O primeiro termo do lado direito de (1) representa a contribuiçăo do campo incidente, cujo efeito, na regiāo iluminada do cilindro, é igual ao que seria fornecido pela aproximaçāo da ótica física [11]. Observa-se, entretanto, que este termo está igualmente presente na regiāo de sombra, na qual a ótica física prescreve correntes nulas. O segundo termo (termo integral) do lado direito de (1) representa a influência das correntes nos demais pontos do contorno sobre o ponto de observação. Para comprimentos de onda muito menores que o diâmetro médio do obstáculo, este termo tende a cancelar os efeitos do campo incidente na regiāo de sombra, introduzindo, apenas, uma pequena correção nos mesmos na regiāo iluminada.

As equaçōes integrais do campo magnético no domínio do tempo (EICM-DT) podem ser obtidas a partir de suas correspondentes (1) e (2) no domínio da frequência, através de transformadas inversas de Fourier

$$
\begin{aligned}
& J(r, t)=\zeta T H^{\text {in }}(\mathbf{r}, \mathbf{t})+ \\
& \frac{T}{2 \pi c} \oint_{C} \int_{0}^{t-\tau} \frac{(\hat{\eta} \cdot \hat{p}) d t^{\prime}}{\left[\left(t-t^{\prime}\right)^{2-\tau^{2}}\right]^{1 / 2}}\left[\frac{J\left(r^{\prime}, t^{\prime}\right)}{\left(t-t^{\prime}+\tau\right)}+\frac{\partial J}{\partial t^{\prime}}\left(r^{\prime}, t^{\prime}\right)\right] d l^{\prime}
\end{aligned}
$$

e

$$
H^{(i n)}(r, t)=n_{x} \frac{G}{\sqrt{\pi}} e^{-G^{2}\left[t+\frac{\left(x-x_{0}\right)}{c}\right]^{2}}
$$

Nestas expressōes, $\tau=\left|\mathbf{r}-\mathbf{r}^{\prime}\right| / \mathbf{c}$, mantendo os demais termos seus significados. Pode-se observar em (3) que a integral no tempo apresenta uma singularidade integrável no seu limite superior. Foi suposto, ainda, que $J(r, t)=0$ para $t<0$.

\subsection{Expressöes para o Campo Distante}

No domínio da frequência, cada componente do campo magnético espalhado, para pontos de observação afastados do cilindro, é determinada, em funçáo da respectiva (para o mesmo modo) componente da densidade de corrente, através da equação 


$$
H^{s}(\mathbf{r}, \omega)=\sqrt{\frac{\omega}{8 \pi c}} \frac{e^{i\left(\frac{\omega}{4}-\frac{\omega}{c} \mathbf{r}_{\mathbf{s}}\right)}}{\sqrt{\mathbf{r}_{\mathbf{s}}}} \oint_{C} \gamma e^{i \frac{\omega}{c}\left(\hat{\mathbf{r}}_{\mathbf{s}} \cdot \mathbf{r}\right)} J\left(\mathbf{r}^{\prime}, \omega\right) d l^{\prime}
$$

Analogamente ao comentário que segue (1), o modo TE $\{T M\}$ associa a componente transversal \{axial\} da densidade de corrente à componente axial \{transversal\} do çampo magnético espalhado. Em (5), $\mathbf{r}_{\mathbf{s}}$ representa o ponto de obseryaçāo, $\mathbf{r}_{\mathbf{s}} \circ$ vetor unitário nesta direçāo e $\mathbf{r}_{\mathbf{s}}=\left|\mathbf{r}_{\mathbf{s}}\right|$. Adicionalmente, $\gamma=-\hat{n}^{\prime} . \hat{r}_{\mathbf{s}}\{1\}$ para o modo TE $\{T M\}$, tendo os demais termos sido já definidos.

A transformada inversa de Fourier de (5) fornece, para o domínio do tempo, a seguinte expressāo

$$
H^{s}\left(\mathbf{r}_{s}{ }^{\prime}, t\right)=\frac{1}{2 \pi \sqrt{2 c r_{s}}} \oint_{C} \int_{0}^{t-\tau_{s}} \frac{\gamma}{\left(t-t^{\prime}-\tau_{s}\right)^{1 / 2}} \frac{\partial J}{\partial t^{\prime}}\left(\mathbf{r}^{\prime}, t^{\prime}\right) d t^{\prime} d l^{\prime}
$$

Nesta equaçāo, $\tau_{s}=\left|\mathbf{r}_{\mathbf{s}}-\mathbf{r}^{\prime}\right| / \mathrm{c}$, mantendo-se os significados dos demais parâmetros. Observa-se, novamente, a existência de singularidade integrável no limite superior da integral relativa ao tempo e a condiçăo $H^{s}\left(r_{s}, t\right)=0$ para $t<0$.

\subsection{Solução Numérica da Equaçáo Integral no Domínio da Frequência}

\subsubsection{Determinação das Densidades de Corrente}

Na solução de (1) pelo método dos momentos [11], foram usadas funçōes delta de Dirac como funçōes de teste (colocaçāo) e funçōes de bases subseccionais do tipo pulso retangular para a expansāo da densidade de corrente. Isto é, supôs-se o contorno $\mathrm{C}$ formado por $\mathrm{N}$ elementos $\mathrm{C}_{\mathrm{n}}$ adjacentes centrados nos pontos $r_{n}$ e de extremidades distando $a_{n}$ dos respectivos centros. A densidade de corrente foi, entāo, representada na forma

$$
J(r, \omega)=\sum_{n=1}^{N} J_{n}(\omega) f\left(r-r_{n}\right)
$$


onde

$$
f\left(r-r_{n}\right)=\left\{\begin{array}{ll}
1 & \left|r-r_{n}\right|<a_{n} \\
0 & \left|r-r_{n}\right|>a_{n}
\end{array} \quad n=1, \ldots, N\right.
$$

sendo $J_{n}(\omega), n=1, \ldots, N$ coeficientes a serem determinados. Escolhendo, ainda, $\omega_{n}(\mathbf{r})=\delta\left(\mathbf{r}-\mathbf{r}_{n}\right), n=1, \ldots, N$ para funçōes de teste, substituindo (7) em (1) e realizando o produto escalar do resultado pelas mesmas, obteve-se

$$
J_{n}(\omega)+\sum_{m=1}^{N} U_{n m}(\omega) J_{m}(\omega)=\zeta T H^{i n}\left(r_{n}, \omega\right), \quad n=1, \ldots, N
$$

onde

$$
U_{n m}(\omega)=\frac{i}{4} \frac{\omega}{c} T \int_{C_{m}}\left(\hat{\eta} \cdot \hat{p}_{n}\right) H f^{(2)}\left(\frac{\omega}{c}\left|\mathbf{r}_{n}-\mathbf{r}^{\prime}\right|\right) d l^{\prime}
$$

Nesta expressão, $\hat{p}_{n}=\left(\mathbf{r}_{n}-\mathbf{r}^{\prime}\right) /\left|\mathbf{r}_{n}-\mathbf{r}^{\prime}\right|$ e $\hat{\eta}=\hat{n}^{\prime}\left(\mathbf{r}^{\prime}\right)\left\{\hat{n}\left(\mathbf{r}_{n}\right)\right\}$ para o modo TE $\{\mathrm{TM}\}$.

No cálculo da integral que aparece em (10), duas situaçōes foram consideradas. A primeira $(m=n)$ envolvia apenas argumentos pequenos para a funçăo de Hankel, o que permitiu calculá-la de forma aproximada [13] Supondo que os raios de curvatura $R_{n}$ dos elementos tornam-se constantes à medida que seus comprimentos $\Delta c_{n}$ decrescem e convencionando-se $R_{n}$ positivo \{negativo\} para contornos localmente convexos \{côncavos\}, concluiu-se que $U_{n n}(\omega)= \pm T \Delta C_{n} /\left(4 \pi R_{n}\right)$, sendo 0 sinal positivo \{negativo\} aplicado ao modo TE $\{T M\}$. Na segunda situaçáo $(m \neq n)$, a integral em (10) foi aproximada pelo produto do valor do seu integrando no ponto médio $\mathbf{r}_{m}$ pelo comprimento $\Delta \mathrm{C}_{\mathrm{m}}$ do elemento.

Desta maneira, (10) foi escrita sob a forma de um sistema de equaçóes lineares de ordem $N$ 


$$
\left[Z_{n m}(\omega)\right]\left[J_{m}(\omega)\right]=\left[H_{n}(\omega)\right]
$$

onde os elementos da matriz impedância $\left[Z_{n m}(\omega)\right]$ são dados por

$$
\begin{aligned}
& Z_{n m}(\omega)=1+U_{n n}(\omega)=\left(1 \pm \frac{T \Delta C_{n}}{4 \pi R_{n}}\right) \\
& Z_{n m}(\omega)=U_{n m}(\omega)=\frac{i}{4} T \frac{\omega}{c} \Delta C_{m}\left(\hat{\eta}_{n m} \cdot \hat{p}_{n m}\right) H_{1}^{(2)}\left(\frac{\omega}{c}\left|r_{n}-r_{m}\right|\right)
\end{aligned}
$$

e

$$
H_{n}(\omega)=\xi_{n} T H^{\text {in }}\left(r_{n}, \omega\right)=\xi_{n} T e^{i \frac{\omega}{c}\left(x_{n}-x_{0}\right)} e^{-\left(\frac{\omega}{2 G}\right)^{2}}
$$

sendo $\hat{p}_{n m}=\left(r_{n}-r_{m}\right) /\left|r_{n}-r_{m}\right|$ e $x_{n}$ a componente $x$ do vetor $r_{n}$. Para $\circ$ modo $T E_{\wedge}\{T M\}$, foram usados: (i) o sinal positivo \{negativo\} em (12); (ii) $\eta_{n m}=n\left(r_{m}\right)\left\{n\left(r_{n}\right)\right\}$ em (13); e (iii) $\xi_{n}=-1\left\{n_{x}\left(r_{n}\right)\right\} e m(14)$. Este sistema de equaçóes foi resolvido usando métodos convencionais.

\subsubsection{Determinação do Campo Distante}

Substituindo (7) em (5) e, mais uma vez, aproximando as integrais resultantes pelos produtos dos valores dos integrandos nos pontos médios $r_{n}(n=1, \ldots, N)$ dos elementos do contorno pelos seus respectivos comprimentos $\Delta \mathrm{C}_{\mathrm{n}}$, obteve-se

$$
H^{s}\left(\mathbf{r}_{s}, \omega\right)=\sqrt{\frac{\omega}{8 \pi c}} \frac{e^{i\left(\frac{\omega}{4}-\frac{\omega}{c} r_{s}\right)}}{\sqrt{r_{s}}} \sum_{n=1}^{N} \gamma_{n} e^{i \frac{\omega}{c}\left(\hat{r}_{s} \cdot r_{n}\right)} J_{n}(\omega) \Delta C_{n}
$$

sendo $\gamma_{n}=-\hat{n}\left(r_{n}\right) \cdot r_{s}\{1\}$ para o modo $T E\{T M\}$.

\subsection{Cálculo de Transientes no Domínio da Frequência}

Os resultados no domínio do tempo foram obtidos em $\mathrm{K}$ instantes $t_{k}=k \Delta t, k=0,1, \ldots,(K-1)$, sendo $\Delta t$ suficientemente pequeno para permitir uma representaçāo satisfatória do pulso incidente. Adicionalmente, para 
atender a necessidades específicas da técnica de soluçāo das equaçōes integrais no domínio do tempo, a desigualdade $c \Delta t<\mathrm{C}_{\min }$, onde $\mathrm{C}_{\min }$ é o comprimento do menor elemento do contorno discretizado, foi também imposta a $\Delta \mathrm{t}$. Estes critérios serāo discutidos mais detalhadamente na próxima seçāo.

No cálculo de transientes, os algoritmos apresentados na seçāo anterior foram usados repetidamente para as frequências $k \Delta f$ onde $k=0,1, \ldots,(K-1)$, resultando $\Delta f$ da igualdade $K \Delta t \Delta f=1$ [14]. Para se calcular, no domínio do tempo, a densidade de corrente no elemento $C_{n}$ do contorno (centrado no ponto $r_{n}$ ), obteve-se a transformada inversa discreta de Fourier da série $J\left(r_{n}, \omega_{k}\right), k=0,1, \ldots,(K-1)$, onde $\omega_{k}=2 \pi k \Delta f$. Analogamente, obteve-se o campo magnético transiente espalhado no ponto $\mathbf{r}_{\mathbf{s}}$. Desejando-se usar a transformada rápida de Fourier (FFT) [14] nestas operaçōes, usou-se $K=2^{Q}$ ( $Q$ inteiro).

\subsection{Soluçāo Numérica da Equaçāo Integral no Domínio do Tempo}

\subsubsection{Determinação das Densidades de Corrente}

A partir de (1), conclui-se que, no domínio da frequência, a densidade de corrente $\mathrm{J}(\mathbf{r}, \omega)$ só depende de variáveis calculadas na mesma frequência $\omega$. Por outro lado, (3) mostra que, no domínio do tempo, a relaçāo entre $J(r, t)$ e $J\left(\mathbf{r}^{\prime}, t^{\prime}\right)$ envolve uma convulução relativa ao tempo. $O$ limite superior $(t-\tau)$ da integral correspondente mostra que $J(r, t)$ é afetada pelas densidades de corrente no interior do cone espaço-tempo definido por

$$
\mathrm{t}^{\prime} \leq \mathrm{t}-\tau \quad \therefore\left|\mathbf{r}-\mathbf{r}^{\prime}\right| \leq \mathrm{c}\left(\mathrm{t}-\mathrm{t}^{\prime}\right)
$$

e delimitado pelas linhas inclinadas da Fig. 2. Consequentemente, na solução de (3) pelo método dos momentos, as funçōes de expansāo e teste que, no domínio da frequência, dependiam apenas de uma variável (r), dependerāo, agora, de duas variáveis ( $r$ e t).

Supôs-se o contorno $\mathrm{C}$ dividido como anteriormente. $\mathrm{O}$ eixo $\mathrm{t}$ foi dividido em intervalos iguais $\left|t-t_{k}\right|<\Delta t / 2$, onde $t_{k}=(k-1 / 2) \Delta t, k=1,2, \ldots$. Desta forma, os interiores dos cones espaço-tempo ficaram discretizados conforme mostra a Fig. 2, no caso do elemento de observaçāo $\left(\mathbf{r}_{n}, t_{k}\right)$, duplamente hachurado. Duas malhas (uniformes, por simplicidade) foram mostradas: no 


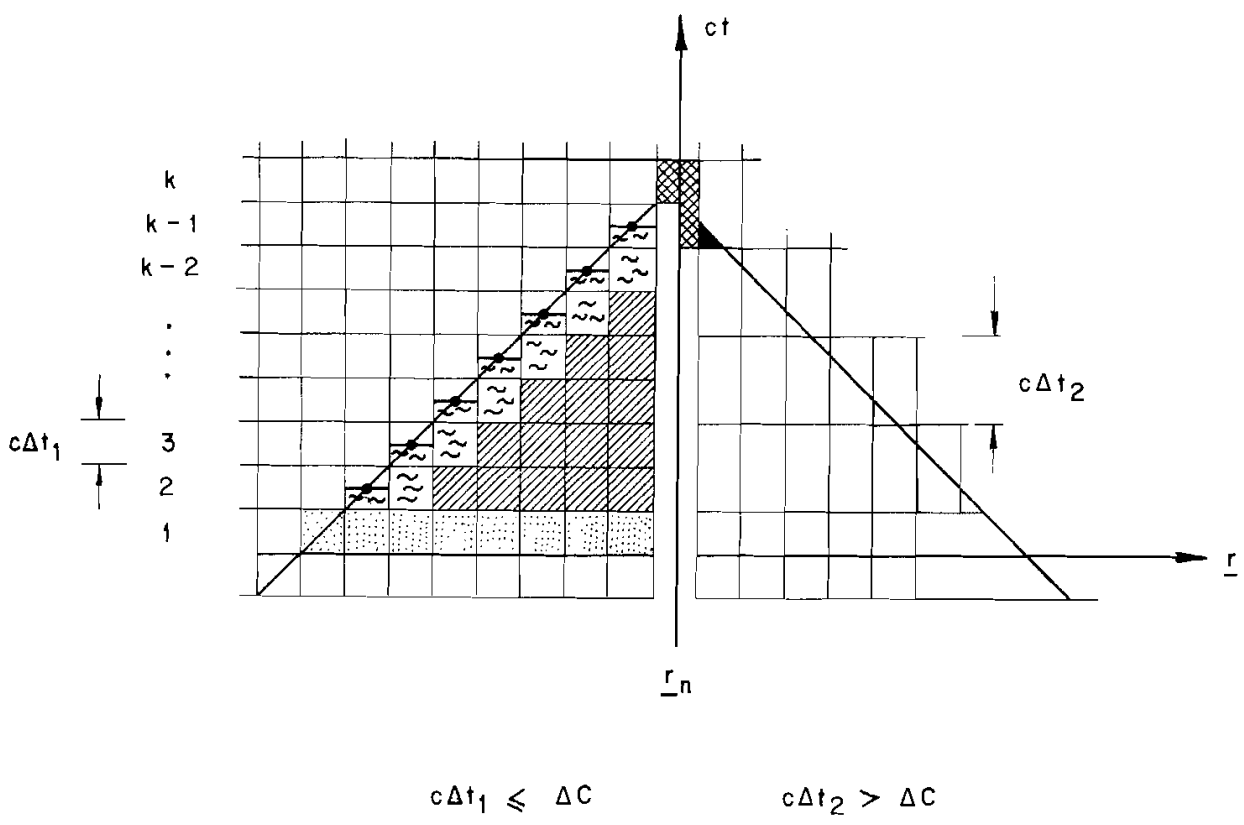

Figura 2. Representação esquemática do cone espaço-tempo para o elemento $\left(r_{n}, t_{k}\right)$ e dos limites para a integraçāo no tempo.

lado esquerdo \{direito do eixo ct, tem-se $c \Delta \mathrm{t}_{1}<\Delta \mathrm{C}\left\{\mathrm{c} \Delta \mathrm{t}_{2}>\Delta \mathrm{C}\right\}$, onde $\Delta \mathrm{C}$ é o comprimento dos elementos do contorno $\mathrm{C}$.

Como resultado da discretizaçāo pela malha 2, a densidade de corrente no elemento de observaçāo depende das densidades de corrente nos elementos vizinhos, no mesmo instante de tempo (triângulo em negrito). Embora esta dependência (introduzida artificialmente) possa ser considerada corretamente, a soluçáo de (3) envolveria a inversāo de matrizes. Por outro lado, a malha 1 nāo introduz esta dependência. Neste caso, altamente desejável, a densidade de corrente no elemento $\left(\mathbf{r}_{n}, t_{k}\right)$ depende apenas das densidades de corrente nos instantes de tempo anteriores (elementos situados no interior do cone espaço-tempo definido por este vértice). Pode, desta forma, ser obtida independentemente das demais densidades de corrente no mesmo instante de tempo, através de um problema de valor inicial, no qual: ( $)$ as densidades de corrente nos elementos $\left(r_{n}, t_{k}\right)$ se anulam para quaisquer valores de $n$ quando $t_{k}$ torna-se negativo ou nulo; (ii) no instante inicial 
(elementos pontilhados na Fig. 2), as densidades de corrente são dadas pelo primeiro termo do lado direito de (3).

Será visto a seguir que o algoritmo proposto por Bennet e Weeks [12] pode ser obtido através do método dos momentos. Para funçōes de teste, foram escolhidas $w_{n k}(r, t)=\delta\left(r-r_{n}\right) \delta\left(t-t_{k}\right), n=1, \ldots, N ; k=2,3, \ldots$ (colocaçáo). As funçōes de expansāo foram definidas pelo produto de duas outras. A primeira, referente à posição do elemento $C_{m}$ no contorno, foi caracterizada por (8). A segunda caracteriza uma variaçāo contínua em relaçāo ao tempo, näo somente no interior de cada elemento como através de suas fronteiras. Sua expressāo funcional, além de depender do elemento $C_{m}$ considerado, foi condicionada ao elemento $\left(\mathbf{r}_{n}, t_{k}\right)$ cuja densidade de corrente estava sendo calculada, conforme será visto brevemente. Para $(m=n)$ (elementos não mostrados na Fig. 2), o tratamento apresentado por Bennet e Weeks [12], análogo ao descrito na subseção 2.3, forneceu um resultado idêntico ao expresso por (12). Para $m \neq n$, cada integral de linha foi aproximada pelo produto do valor do integrando no ponto $r_{m}$ pelo comprimento do elemento $\mathrm{C}_{\mathrm{m}}$. Desta forma, obteve-se

$J\left(\mathbf{r}_{n}, t_{k}\right)=\left(1 \pm \frac{T \Delta C_{n}}{4 \pi R_{n}}\right)^{-1}\left[\mp T H^{i n}\left(r_{n}, t_{k}\right)+\frac{T}{2 \pi c} \sum_{m=1}^{N} F_{n k}\left(r_{m}\right) \Delta C_{m}\right]$

$m \neq n$

onde $n=1, \ldots, N, k=2,3, \ldots$, os sinais superiores \{inferiores $\}$ são usados para o modo TE $\{$ TM $\}$,

$$
H_{\text {in }}\left(r_{n}, t_{k}\right)=n_{x} \frac{G}{\sqrt{\pi}} e^{-G^{2}\left[t_{k}+\frac{\left(x_{n}-x_{0}\right)}{c}\right]^{2}}
$$

e

$F_{n k}\left(r_{m}\right)=\left(\hat{\eta}_{n m} \cdot \hat{p}_{n m}\right) \int_{0}^{t_{k}-\tau_{n m}} \frac{d t^{\prime}}{\left[\left(t_{k}-t^{\prime}\right)^{2}-\tau_{n m}^{2}\right]^{2}}\left[\frac{J\left(r_{m}, t^{\prime}\right)}{\left(t_{k}-t^{\prime}+\tau_{n m}\right)}+\frac{\partial J}{\partial t^{\prime}}\left(\mathbf{r}_{m}, t^{\prime}\right)\right]($

Nesta expressão, $\tau_{n m}=\left|\mathbf{r}_{n}-\mathbf{r}_{m}\right| / \mathbf{c}$, tendo os demais termos sido definidos anteriormente. Finalmente, dois tipos de funçāo relativas ao tempo foram 
usadas para completar a caracterização das funçōes de expansão dos elementos $C_{m} \neq C_{n}$. Para os elementos hachurados, pontilhados ou em branco na Fig. 2, foram usadas funçōes que permitiram a integração de (19) pela regra de Simpson [13] no intervalo de tempo correspondente. Para os elementos ondulados na Fig. 2, polinômios do quarto grau (ou inferior) em t foram ajustados aos valores conhecidos das densidades de corrente nas vizinhanças do limite do cone espaço-tempo. Desta forma, a integral em (19) foi calculada analiticamente no restante do intervalo de tempo.

\subsubsection{Determinação do Campo Distante}

Utilizando-se um tratamento idêntico ao da subseçāo anterior, (6) transforma-se em

$$
H^{s}\left(r_{s}, t_{k}\right)=\frac{1}{2 \pi \sqrt{2 c r_{s}}}=\sum_{m=1}^{N} H_{s k}\left(r_{m}\right) \Delta C_{m}
$$

onde

$$
H_{s k}\left(\mathbf{r}_{m}\right)=\gamma_{m} \int_{0}^{t_{k}-\tau_{s m}} \frac{1}{\left(t_{k}-t^{\prime}-\tau_{s m}\right)^{1 / 2}} \frac{\partial J}{\partial t^{\prime}}\left(r_{m}, t^{\prime}\right) d t^{\prime}
$$

sendo $\gamma_{m}=-\hat{n}\left(r_{m}\right) \cdot \hat{p}_{s m}\{1\}$ para o modo $T\{T M\}$. Tem-se, ainda, que $\hat{p}_{s m}$ é o vetor unitário na direçāo $\left(r_{s}-r_{m}\right)$ e $\tau_{s m}=\left|r_{s}-r_{m}\right| / c$. A integração em (21) é idêntica àquela em (19).

\section{Técnicas de Redução do Tempo de Processamento no Domínio da Frequência}

Foi dito que uma parcela substancial do tempo necessário para a determinação, no domínio da frequência, dos campos transientes espelhados por uma estrutura qualquer, se deve aos cálculos de todos os elementos das matrizes impedância em um grande número de frequências igualmente espaçadas. De acordo com (13), este cálculo envolve a função de Hankel do segundo tipo e primeira ordem. Esta pode ser obtida, com boa precisāo, com o auxílio de aproximaçōes polinomiais [13]. Sendo os polinômios interpolantes do 
sexto grau, o tempo de processamento seria elevado para a aplicaçāo pretendida.

Nesta seçāo, serāo apresentadas técnicas utilizadas no domínio da frequência para a redução do tempo de cálculo dos elementos da matriz impedância.

\section{1. Simetria dos Campos e Correntes e Variaçào da Potência do Pulso Incidente}

Os elementos $s_{k}(k=0,1, \ldots, k-1)$ de uma série temporal são reais se e somente se os elementos $S_{k}(k=0,1, \ldots, K-1)$ de sua transformada discreta de Fourier apresentam a simetria $S_{k-k}=S_{k}^{*}$ (onde $S_{k}^{*}$ representa 0 complexo conjugado de $S_{k}$ ) [12]. Este teorema apresenta uma condiçāo necessária e suficiente para que cálculos realizados no domínio da frequência forneçam sinais reais no domínio do tempo. Implicitamente, indica que, para se determinar $\mathrm{K}$ valores de uma série temporal real, é suficiente determinarem-se as primeiras $(K / 2)+1$ amostras no domínio da frequência, reduzindo o tempo de processamento à metade do estimado inicialmente.

Por outro lado, (2) mostra que a intensidade do campo incidente diminui rapidamente com a frequência. Parece, então, razoável supor que as contribuiçóes dos harmônicos da frequência $\Delta$ f superiores a um dado limiar possam ser desprezadas, na presença da soma das contribuiçóes de todos os harmônicos inferiores ao mesmo. Para tanto, é nacessário que esta soma contenha uma alta percentagem da potência do pulso incidente. A partir de (2), obtém-se que os cálculos precisam ser realizados apenas para os harmônicos da frequência $\Delta f$ inferiores a $k_{\max }$, onde

$$
2 \pi k_{\max } \Delta f / G=5 \therefore k_{\max }=0.8 \mathrm{G} / \Delta f
$$

Para os valores típicos usados neste trabalho $\left(G=6 \times 10^{8} \mathrm{~s}^{-1} \mathrm{e}\right.$ $\Delta f=23,8 \mathrm{MHz}$ ), obtém-se $k_{\max }=20$.

\section{2. Interpolaçáo}

Recentemente, Newman [15] observou que os elementos das matrizes impedância obtidas através do método dos momentos, em muitos casos, variam suavemente com a frequência. Em seguida, associou o cálculo efetivo dos valores de um dado elemento em frequências espaçadas de um múltiplo 
elevado $\left(k_{0}\right)$ da frequência $\Delta f$ com a interpolação de polinômios (no caso, do segundo grau) através dos mesmos. Desta forma, obteve valores do mesmo elemento em frequências intermediárias. Como resultado da aplicação desta técnica, os tempos de processamento foram reduzidos de quatro a oito vezes nos exemplos apresentados.

É essencial para o sucesso da técnica que a variação com a frequência dos elementos da matriz impedância seja bem representada pelo polinômio interpolante. O seguinte critério foi apresentado para a seleçāo do maior valor de $k_{0}$ ainda capaz de assegurar a qualidade dos resultados

$$
k_{0}=\frac{1}{4} \frac{c}{L \Delta f}=\frac{1}{4}\left(\frac{\Delta \lambda}{L}\right)
$$

onde $L$ é o diâmetro médio da estrutura analisada e $\Delta \lambda=c / \Delta f$. Observa-se, portanto, que a técnica é mais eficiente ( $k_{0}$ grande) para estruturas pequenas em relaçáo a $\Delta \lambda$. As dificuldades decorrentes da aplicação da téenica de interpolaçāo a estruturas de dimensōes maiores serāo observadas na subseção 4.1. Naquela subseção, será utilizado um algoritmo híbrido, desenvolvido para assegurar a qualidade dos resultados finais. Inicialmente, os elementos das matrizes correspondentes aos extremos e ao centro da faixa de frequências em estudo são calculados, usando-se (13). Em seguida, calcula-se para cada elemento das matrizes, o parâmetro

$$
P=2 \frac{k_{0} \Delta f}{c}\left|r_{n}-r_{m}\right|
$$

que representa a variação máxima do argumento da funçāo de Hankel na faixa considerada. Quando este parâmetro não ultrapassa um determinado limiar (no caso, 0,38), a interpolaçāo é possível. Caso contrário, (13) é utilizada diretamente.

\subsection{Consulta a uma Tabela de Funçōes de Hankel}

Os elementos da matriz impedância em (13) podem ser representados em termos do produto $\left[u H^{(2)}(u)\right]$, onde $u=\omega\left|r_{n}-r_{m}\right| / c$. A função entre colchetes foi tabelada. Isto é, armazenaram-se, num vetor complexo, os valores daquela funçāo para os argumentos reais $u_{i}=u_{0}+(i-1) \delta u_{\text {, }}$ $i=1, \ldots$, l. 
Dados $n, m$ e w e calculado o valor de $u$, o cálculo do termo entre colchetes e do elemento correspondente da matriz impedância torna-se extremamente simples, a partir da interpolaçāo entre os elementos da tabela mais próximos do argumento obtido. A precisāo dos resultados dependerá da resoluçāo da tabela (que, associada ao seu domínio de validade, define a área necessária para seu armazenamento) e da técnica de interpolaçāo usada no cálculo de valores intermediários. No caso, os valores $u_{0}=0,5, \delta u=1 / 60$, e $I=3810$, juntamente com a interpolaçáo linear entre elementos da tabela, foram usados.

\section{Resultados}

\subsection{Comparaçāo Entre Tempos de Processamento}

Para mostrar, através de um exemplo, a importância das técnicas descritas na seçāo anterior, os campos transientes espalhados por um cilindro circular de raio igual a $1 \mathrm{~m}$ foram calculados em três direçōes $\left(\theta=0^{\circ}, 90^{\circ}\right.$ e $180^{\circ}$, conforme mostra a Fig. 1), no domínio da frequência. O contorno do cilindro foi dividido em 51 partes iguais, sendo o pulso incidente caracterizado por $G=6 \times 10^{8} \mathrm{~s}^{-1}$. Os campos espalhados foram calculados em 128 instantes de tempo, sendo $\Delta t=0,328 \mathrm{~ns}$. Nestes cálculos, somente as primeiras 26 frequências harmônicas de $\Delta f=23,80 \mathrm{MHz}$ (incluindo a componente $\mathrm{DC}$ ) foram utilizadas.

\begin{tabular}{lcccc}
\hline & TESTE 1 & TESTE 2 & TESTE 3 & TESTE 4 \\
\hline $\mathrm{u}$ & $0,5<\mathrm{u}<64$ & $0,5<\mathrm{u}<64$ & $0,5<\mathrm{u}<20$ & $0,5<\mathrm{u}<64$ \\
$\mathrm{P}$ & 0,25 & 0,38 & 0,25 & 10 \\
KiNT & 0 & 2.550 & 0 & 63.750 \\
KTAB & 63.750 & 61.200 & 57.732 & 0 \\
KSUB & 0 & 0 & 6.018 & 0 \\
Tempo de CPU (s) & 56,16 & 56,66 & 77,25 & 86,22 \\
\hline
\end{tabular}

Tabela 1. Comparação entre tempos de processamento no domínio da frequência (CDC Cyber 170/835).

Os resultados obtidos foram resumidos na Tabela 1. Na primeira linha, apresentaram-se os limites utilizados na construção da tabela de valores de 
$\left[\mathrm{uH}_{\mathrm{l}}^{(2)}(\mathrm{u})\right]$. Em seguida, observam-se os valores do parâmetro $P$ definido em (24). Ainda na Tabela 1, K $\mathrm{INT}_{\mathrm{N}}, \mathrm{K}_{\mathrm{TAB}}$ e $\mathrm{K}_{\text {SUB }}$ representam os números de vezes que a técnica de interpolação, a tabela de valores de $\left[\mathrm{uH}_{1}^{(2)}(\mathrm{u})\right]$ e subrotinas especiais (aproximaçōes polinomiais para as funçōes de Hankel) foram respectivamente usadas, em cada teste, nos cálculos dos elementos das matrizes impedância.

O primeiro teste foi projetado para assegurar a utilizaçāo da tabela em todos os casos. No segundo teste, o valor de $P$ foi aumentado, para permitir o uso da interpolaçāo (assegurada a qualidade dos resultados). No terceiro teste, forçou-se uma utilização maior das subrotinas especiais. Finalmente, a técnica da interpolaçāo foi utilizada em todos os casos no quarto teste, mesmo que em detrimento da qualidade dos resultados.

Os tempos de processamento no computador CDC Cyber 170/835 são observados na última linha da Tabela 1. As soluçōes do problema nos domínios do tempo e da frequência (utilizando sempre as subrotinas especiais) foram obtidas em $2.353,5 \mathrm{~s}$ e $751,1 \mathrm{~s}$, respectivamente. Deve-se ressaltar, ainda, que os resultados obtidos para o campo espalhado nos três primeiros testes mostraram-se aceitavelmente próximos dos obtidos pelas técnicas (mais exatas) apresentadas imediatamente acima. No quarto teste, um resultado inaceitável já era esperado, uma vez que sempre aproximou-se a funçăo de Hankel por uma parábola, mesmo que os três pontos envolvidos estivessem substancialmente afastados.

Estes resultados mostram, inicialmente, ser preferivel realizar o cálculo de transientes espalhados por estruturas cilíndricas no domínio da frequência, mesmo que técnicas de reduçāo do tempo de processamento nāo sejam utilizadas. Mostram, ainda, que estas podem ser introduzidas no domínio da frequência, sem qualquer aumento significativo no ruído numérico, mesmo quando acompanhadas do truncamento dos cálculos relativos aos harmônicos elevados de f. Por outro lado, uma comparação entre os tempos de CPU dos testes 1, 2 e 4 mostra que o uso da tabela de funçóes de Hankel torna o processamento mais rápido que o emprego da técnica de interpolaçāo. Sendo a tabela construída com uma resolução maior, seu uso introduz erros inferiores aos causados pela técnica da interpolaçăo. De fato, o segundo teste mostra que esta raramente é usada quando se deseja manter a qualidade dos resultados. 


\subsection{Exemplos}

Na Fig. 3, representam-se os campos magnéticos transientes espalhados (modos TE e TM) por um cilindro circular de raio igual a $1 \mathrm{~m}$. Na Fig. 4 apresentam-se os campos transientes espalhados (modo TE) por um cilindro de seção reta composta por uma parte central retangular (comprimento 1,8 $\mathrm{m}$ ) e extremos semi-circulares (raios iguais a $0,6 \mathrm{~m}$ ), para incidências frontal e lateral. Finalmente, a Fig. 5 mostra os campos transientes espalhados (modo TE) por um cilindro de seçāo reta na forma de um refletor de canto de arestas e vértices suavizados de comprimento e largura respectivamente
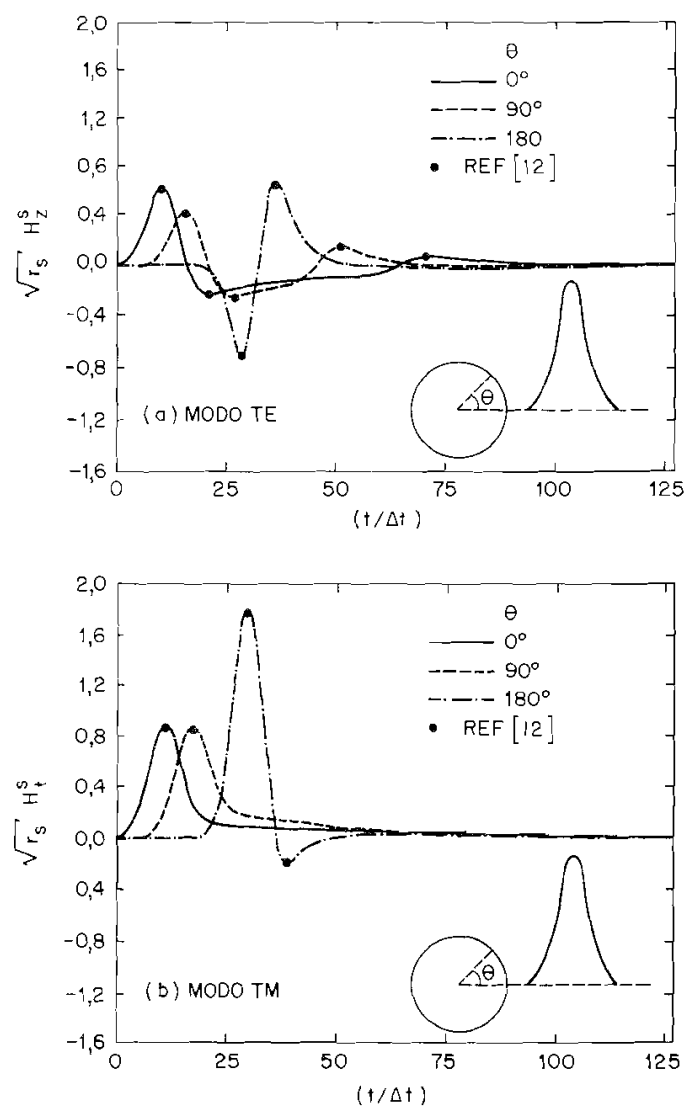

Figura 3. Campos magnéticos espalhados pelo cilindro circular. Além das normalizaçōes indicadas, as amplitudes e direçōes dos campos espalhados se referem às do pulso incidente. As duraçōes de todos os campos (incidente e espalhado) e, através da transformação $n_{d}=d /(c \Delta t)$, as dimensōes do espalhador, se referem à escala horizontal. 

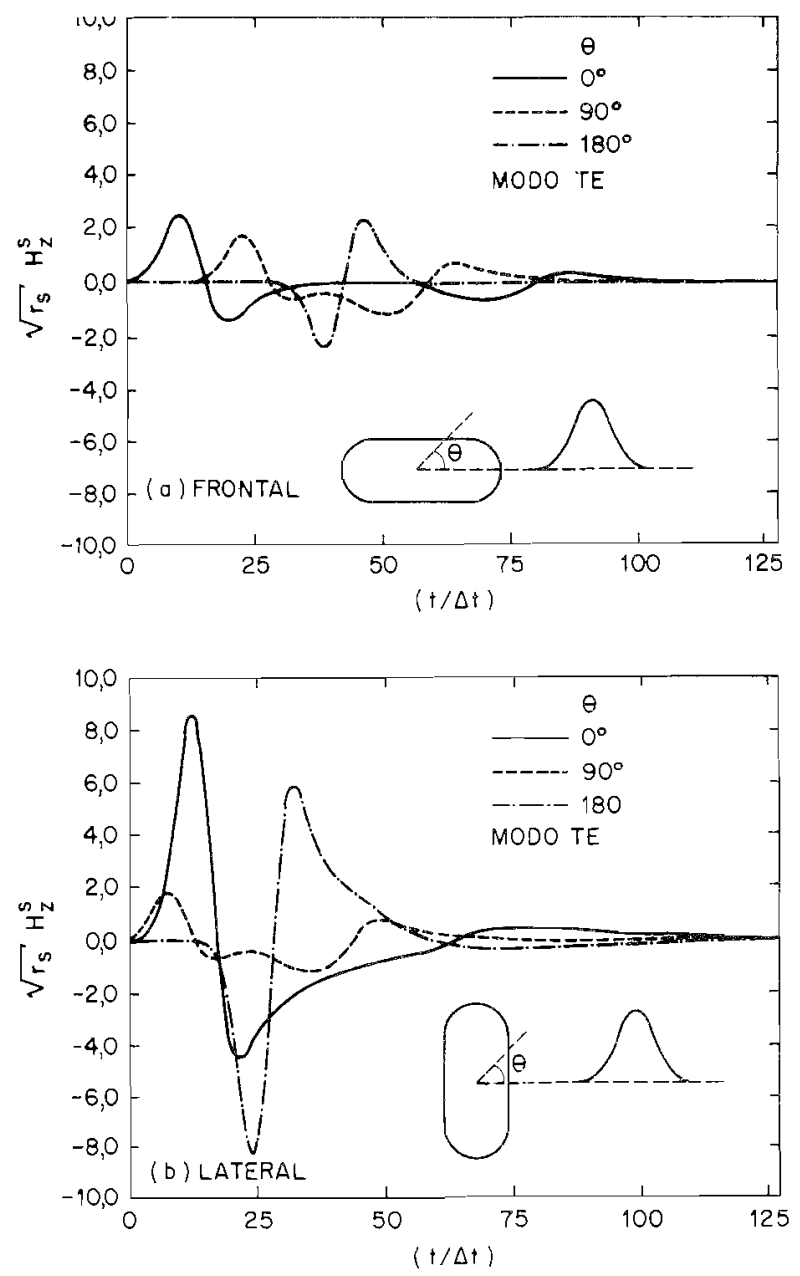

Figura 4. Campos magnéticos espalhados pelo cilindro de seçāo reta composta, com incidência: (a) frontal; (b) lateral.

As mesmas normalizaçōes descritas na Fig. 3 foram utilizadas.

iguais a 2,79 $\mathrm{m}$ e $0,5 \mathrm{~m}$. Em todos os casos, os campos espalhados foram observados nas direçōes $0^{\circ}, 90^{\circ}$ e $180^{\circ}$ (relativas à direçāo de incidência). Nestas figuras, as amplitudes do pulso incidente (caracterizado por $\mathrm{G}=6 \times 10^{8} \mathrm{~s}^{-1}$ ) foram apresentadas em valores das respectivas escalas verticais, sendo suas duraçōes, em conformidade com a escala horizontal (comum) utilizada, normalizadas em relaçáo a $\Delta t=0,328 \mathrm{~ns}$. Cada comprimento $d$ de cada seçāo reta foi convertido para a mesma escala através da

Revista da Sociedade Brasileira de Telecomunicações 
relaçäo $n_{d}=d /(c \Delta t)$. Por outro lado, os contornos foram respectivamente divididos em 51, 64 e 50 elementos de mesmo comprimento.

Os resultados de [12] foram representados por círculos na Fig. 3. As diferenças existentes nāo podem ser observadas na escala utilizada. $\mathrm{Na}$ ausência de informaçōes completas, evitaram-se as comparaçōes entre os resultados relativos aos demais casos. Obsenvam-se, entretanto, boas concordâncias entre as formas gerais dos respectivos campos transientes espalhados.

Em todos os exemplos apresentados, os primeiros pulsos observados nas três direçōes resultam, predominantemente, das correntes induzidas diretamenie pelo campo incidente. Na regiāo iluminada do cilindro, estes pulsos podem ser interpretados como os resultados da reflexáo especular. Em seguida, observam-se, nas três direçōes, contribuiçōes das correntes originadas das interaçōes mútuas entre os diversos elementos do contorno. Estas começam a atuar imediatamente após a interaçāo direta entre o pulso incidente a o cilindro, explicando, em parte, as assimetrias existentes entre as partes anteriores e posteriores das componentes iniciais dos campos espalhados. Os efeitos das correntes resultantes das interaçōes mútuas predominam a partir do momento em que cessa a interaçāo direta já mencionada. Os últimos picos observados nas diversas curvas podem ser interpretados como contribuiçóes de "creeping waves". O efeito máximo destas ondas ocorre na direçăo $180^{\circ}$, em decorrência das simetrias existentes.

Uma comparação entre as figuras 3(a) e 3(b) mostra os efeitos da polarizaçāo do campo incidente sobre o resultado. Em particular, as amplitudes rnáximas dos pulsos dianteiros do modo TM sāo superiores às do modo TE. Ass componentes resultantes da interaçāomútua entre dois elementos do cilindro, por outro lado, têm duraçāo maior e são menos pronunciadas no modo TM, näo causando, em geral, inversões no sinal do campo espalhado.

Observam-se, em todas as figuras, retardos e avanços dos campos espalhados nas demais direçōes, em relaçāo àquele espalhado na direçāo $0^{\circ}$. Por exemplo, para a incidência frontal mostrada na Fig. 4(a), a distância entre o elemento do contorno excitado inicialmente pelo campo incidente e o ponto de observação na direçāo $90^{\circ}$ é superior à correspondente na direção $0^{\circ}$. O campo espalhado na direçāo $90^{\circ}$ encontra-se, portanto, nitidamente atrasado em relaçāo ao observado na direçāo de referência. Por outro lado, para a 


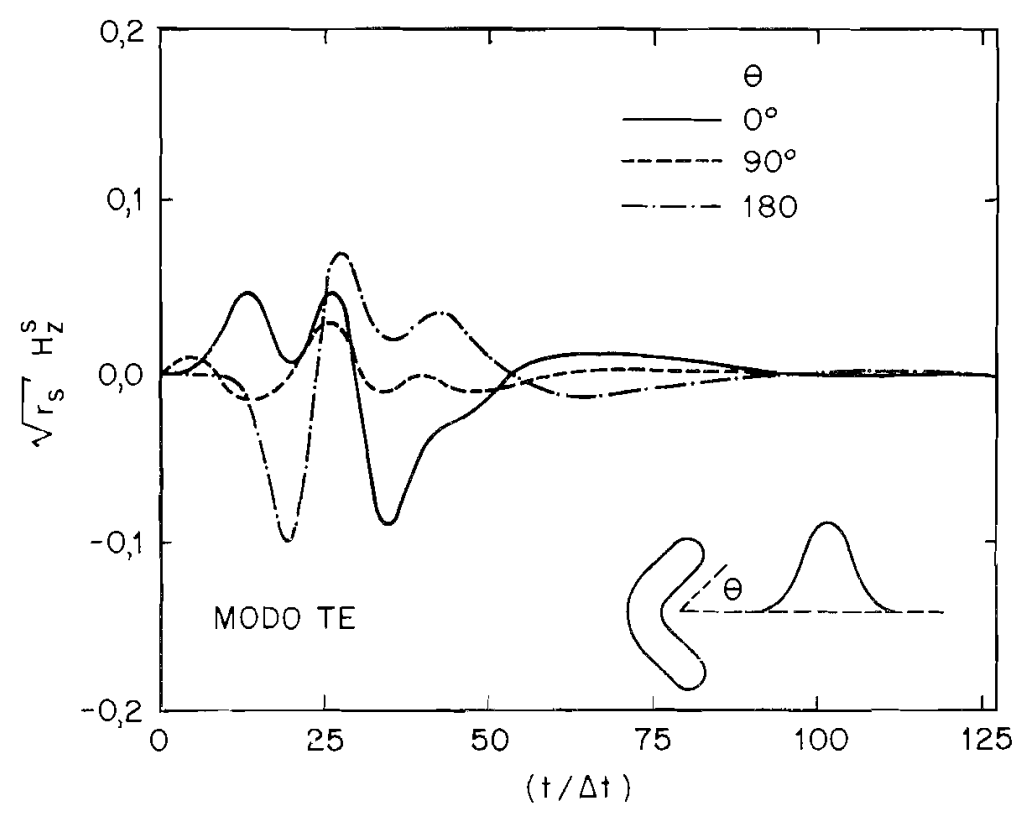

Figura 5. Campos magnéticos espalhados pelo refletor de canto. As mesmas normalizaçōes descritas na Fig. 3 foram utilizadas.

incidência lateral mostrada na Fig. 4(b), a distância entre o canto superior da face plana ao ponto de observaçáo da direçāo $90^{\circ}$ é menor que a distância entre o centro da mesma face e o ponto de observaçāo da direçāo $0^{\circ}$. Por conseguinte, o campo espalhado na direçáo $90^{\circ}$ apresenta-se ligeiramente adiantado em relação ao espalhado na direção $0^{\circ}$.

Esta estrutura permite a observaçāo de características especiais. Para ambas as incidências, as formas de onda relativas ao espalhamento na direção $90^{\circ}$, sāo idênticas. Este resultado era esperado, em consequência do teorema da reciprocidade [1]. Obsenva-se, na Fig. 4(a), que o campo espalhado na direçāo $0^{\circ}$ se anula no intervalo de tempo compreendido entre os dois pulsos negativos, correspondente àquele no qual o pulso incidente se propaga na regiāo uniforme (retângulo) do cilindro. As variaçōes das seçōes yz ( $x$ constante) na parte posterior do cilindro sāo responsáveis pelo segundo pulso negativo observado na mesma curva.

Finalmente, observa-se na Fig. 5, além daquelas já apresentadas, outra componente dos campos espalhados pelo refletor de canto, resultante das 
reflexōes mútíplas que ocorrem na regiāo côncava, iluminada no presente caso. O segundo pulso positivo observado em cada direçáo resulta deste mecanismo.

\section{Conclusóes}

Observou-se que o tempo necessário ao cálculo dos campos transientes espalhados por um cilindro infinito condutor perfeito de seção reta arbitrária é sempre maior no domínio do tempo que no da frequência, mesmo que nemhuma das técnicas descritas na Seçăo 3 seja usada. Isto pode ser Usitícado pela presença, no domínio do tempo, da operaçāo convoluçāo entre o núcleo do operador integral e a densidade de corrente que se deseja determinar, ao passo que, no domínio da frequência, tem-se, simplesmente, o produto de suas transformadas de Fourier.

Esta conclusáo não deve ser estendida imediatamente para o problema tridimensional. Neste caso, o núcleo do operador integral é a funçāo delta de Dirac, de modo que aquela convolução pode ser realizada trivialmente.

Uma tabeia de funçōes foi utilizada nos cálculos dos elementos das matrizes impedância. Esta técnica, em conjunto com o truncamento dos cálculos em irequências elevadas, produziu as maiores reduçōes no tempo de processamento. Adicionalmente, apresenta as seguintes vantagens em relaçāo à técnica de interpolação: precisāo uniformemente satisfatória em toda a faixa de utilizaçäo e necessidade de área de armazenamento relativamente pequena, capaz de compartilhar espaços prévia e obrigatoriamente alocados.

Finalmente, os campos transientes espalhados por três estruturas foram anailisados. Os resultados obtidos mostraram boas concordâncias com os apresentados na literatura [9] e [12].

\section{Agradecimentos}

Este trabalho foi realizado no período de vigência do convênio Finep PUC/RIO n 4.3.89.0207-89/90. Durante o mesmo, E. Costa recebeu Bolsa de Pesquisa do CNPq e C. L. S. Souza Sobrinho recebeu Bolsa de Mestrado da CAPES (PICD). A idéia da construçāo de uma tabela, apresentada na 
Seçāo 3, foi lembrada, num contexto diferente, pelo Prof. Raimundo Sampaio Neto, a quem os autores expressam seus agradecimentos.

\section{Referências}

[1] J. Van Bladel, "Electromagnetic Fields", McGraw-Hill, New York, 1964.

[2] S. Silver, "Microwave Antenna Theory and Design", McGraw-Hill, New York, 1949.

[3] J.B. Keller, "Geometrical Theory of Diffraction", Journal of the Optical Society of America, vol. 52, n² 2, Fevereiro 1982, pp. 116-130.

[4] A. Taflove e K.R. Umashankar, "The Finite-Difference Time Domain (FD-TD) Method for Electromagnetic Scattering and Interaction Problems", Journal of Electromagnetic Waves and Applications, vol. 1, no 3, 1987, pp. 243-267.

[5] A. Taflove e K.R. Umashankar, "Review of FD-TD Numerical Modeling of Electromagnetic Wave Scattering and Radar Cross Section", Proceedings of the IEEE, vol. 77, no 5, Maio 1989, pp. 682-699

[6] K.K. Mel e J. Van Bladel, "Scattering by Perfectly-Conducting Rect angular Cylinders", IEEE Transactions on Antennas and Propagation, vol. AP-11, n² 2, Março 1963, pp. 185-193

[7] M.G. Andreasen, "Scattering from Parallel Metallic Cylinders with Arbitrary Cross Sections", IEEE Transactions on Antennas and Propagation, vol. AP-12, $n^{\circ}$ 6, Novembro 1964, pp. 746-754.

[8] A.J. Poggio e E.K. Miller, "Integral Equation Solutions of Three-Dimensional Scattering Problems", in Computer Techniques for Electromagnetics, R. Mittra (ed.), Pergamon Press, New York, 1973.

[9] R. Mittra, "Integral Equation Methods for Transient Scattering, in Transient Electromagnetic Fields", L.B. Felsen (ed.), Topics in Applied Physics, vol. 10, Springer-Verlag, Berlin, 1976.

[10] C.L.S. Souza Sobrinho, "Espalhamento de Transientes Eletromagnéticos por Cilindros Condutores de Seçōes Retas Arbitrárias", Dissertaçāo de Mestrado, Departamento de Engenharia Elétrica, PUC/RIO, Julho de 1989. 
[11] R.F. Harrington, "Field Computation by Moment Methods", Macmillan, New York, 1968.

[12] C.L. Bennet e W.L. Weeks, "Transient Scattering from Conducting Cylinders", IEEE Transactions on Antennas and Propagation, vol. AP18, $n^{9} 5$, Setembro 1970, pp. 627-633.

[13] M. Abramowitz e I.A. Stegun, "Handbook of Mathematical Functions", Dover, New York, 1968.

[14] L.R. Rabiner e B. Gold, "Theory and Applications of Digital Signal Processing", Prentice-Hall, Englewood Cliffs, New Jersey, 1975.

[15] E.H. Newman, "Generation of Wide-Band Data from the Method of Moments by Interpolating the Impedance Matrix", IEEE Transactions on Antennas and Propagation, vol. AP-36, n² 12, Dezembro 1988, pp. 1820-1824.

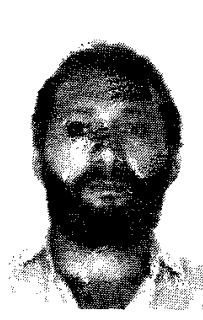

CARLOS LEÔNIDAS S. S. SOBRINHO nasceu em Belém, Pará, em 31 de outubro de 1953. Concluiu o curso de Engenharia Elétrica em 1981 na Universidade Federal do Pará. Obteve o grau de Mestre em Ciências em Engenharia Elétrica na Pontifícia Universidade Católica do Rio de Janeiro em 1989. Está atualmente matriculado no programa de doutorado na Faculdade de Engenharia Elétrica da UNICAMP, na área de Eletromagnetismo Aplicado. Ingressou no quadro docente da Universidade Federal do Pará em 1986, como Professor Auxiliar, e é, desde 1989, Professor Assistente na mesma Universidade.

EMANOEL P. DE OLIVEIRA COSTA formou-se em Engenharia de Telecomunicações no Instituto Militar de Engenharia em 1972 e concluiu o mestrado em Engenharia Elétrica no mesmo Instituto em 1974. Concluiu o doutorado em Engenharia Elétrica na Universidade de Cornell em 1977. Ingressou na PUC/Rio em 1978, onde é atualmente Professor Associado. Desde então, tem estado envolvido em ensino e pesquisa no Centro de Estudos em Telecomunicaçōes da Universidade Católica (CETUC). No perfodo de 1984 a 1986, realizou atividades de pesquisa na área de Propagação lonosférica no Air Force Geophysics Laboratory (Hanscom AFB, MA, Estados Unidos) como Pesquisador Visitante do Emmanuel College (Boston, MA). Seus interesses atuais estão nas áreas de propagação de sinais radioelé tricos em meios naturais e aplicaçōes de métodos numéricos a problemas de eletromagnetismo. 